\title{
The relationship between cardiac endothelium and fibroblasts: it's complicated
}

\author{
Ravi Karra, ${ }^{1,2}$ Agoston 0. Walter, ${ }^{2}$ and Sean M. Wu${ }^{3,4}$ \\ ${ }^{1}$ Regeneration Next and ${ }^{2}$ Department of Medicine, Duke University, Durham, North Carolina, USA. ${ }^{3}$ Cardiovascular Institute and Institute for Stem Cell Biology and Regenerative Medicine \\ and ${ }^{4}$ Department of Medicine, Division of Cardiovascular Medicine, Stanford University, Stanford, California, USA.
}

\begin{abstract}
Coronary revascularization is an effective means of treating ischemic heart disease; however, current therapeutic revascularization strategies are limited to large caliber vessels. Because the mammalian heart scars following cardiac injury, recent work showing that cardiac fibroblasts can transdifferentiate into new coronary endothelium raises a new and exciting approach to promoting endogenous revascularization following cardiac injury. In this issue of the $J C I$, He et al. report on their employment of a battery of lineagetracing tools to address the developmental origins of fibroblasts that give rise to new endothelial cells. Surprisingly, cardiac fibroblasts did not appear to contribute appreciably to regeneration of cardiac endothelium. Instead, cardiac endothelial cells were likely to proliferate and generate new endothelium following injury. As these conclusions diverge from prior findings, additional work will be required to understand the sources that generate cardiac endothelium in new blood vessels after injury. Clarification of the origins of coronary endothelial cells during cardiac repair is essential for identifying improved approaches to revascularizing damaged myocardium in patients with ischemic heart disease.
\end{abstract}

\section{Background}

Ischemic heart disease is the leading cause of cardiovascular death in the US (1). Current revascularization strategies, including percutaneous and surgical interventions, have dramatically improved ischemic heart disease-related mortality. However, these strategies are limited to large epicardial arteries that can be stented or accommodate bypass grafts. Approaches that offer more complete revascularization of smaller arterial beds have the potential for additional improvements in cardiovascular diseaserelated mortality. Accordingly, the basic mechanisms of endogenous revascularization have been intensely investigated.

\section{Origins and fates of the cardiac} endothelium during development In fetal hearts, the emergence of the coro- nary vasculature coincides with thickening of the ventricular wall. Initially, the coronary vasculature develops as an immature plexus, but it is later remodeled into a sophisticated network of vessels that is required for normal cardiac function. Recent work has linked the developmental origins of the coronary endothelium to the endothelium of the sinus venosus and the endocardium and to a subset of epicardial cells (2-5).

While preexisting endothelial cells are the source of the majority of coronary endothelial cells during cardiac development, endocardial cells possess the unique capacity of undergoing endothelial-tomesenchymal transition (EndoMT). In response to local TGF- $\beta$ and Notch signals, endocardial cells become the mesenchyme of the endocardial cushions that

Related Article: p. 2968

Conflict of interest: The authors have declared that no conflict of interest exists

Reference information: / Clin Invest. 2017;127(8):2892-2894. https://doi.org/10.1172/JCI95492.

remodel to form cardiac valves (6). More recently, some endothelial cells, along with epicardial cells, have been shown to contribute to cardiac fibroblast formation in the developing mouse heart (7). Along these lines, coronary endothelium has also been suggested as undergoing EndoMT postnatally and contributing fibroblasts following cardiac pressure overload (8). However, studies with markers more specific to cardiac fibroblasts have suggested that EndoMT following pressure overload is not likely to occur as extensively as previously described (7).

\section{Origins of new cardiac} endothelium following injury In light of the apparent plasticity of endothelial cells in contributing to mesenchymal/fibroblast cells during development and after cardiac injury, Ubil et al. sought to determine whether a mesenchymalto-endothelial transformation (MEndoT) occurs in the adult mouse heart following injury (9). Ubil et al. used a transgenic strain of mouse carrying an inducible Cre recombinase allele under the control of a 6-kb enhancer/promoter of Col1a2 (Col1a2CreER mice). This transgene was previously described as being specifically expressed in fibroblasts (10). Five days after treatment with tamoxifen to label cardiac fibroblasts, Ubil et al. showed that 30\%-40\% of reporter-labeled cells at the injury site expressed endothelial markers within 3 days of injury (Figure 1A). Ubil and colleagues also demonstrated that the observed MEndoT is p53 dependent. The abundance of cardiac fibroblasts with a MEndoT signature accumulating at the site of cardiac injury raised the possibility that new endothelial cells may be readily induced from scar-forming fibroblasts to revascularize the heart after ischemic injury.

In this issue, He et al. report on how they set out to investigate the exact fibroblast subpopulation capable of undergoing MEndoT conversion (11). First, He and col- 
A

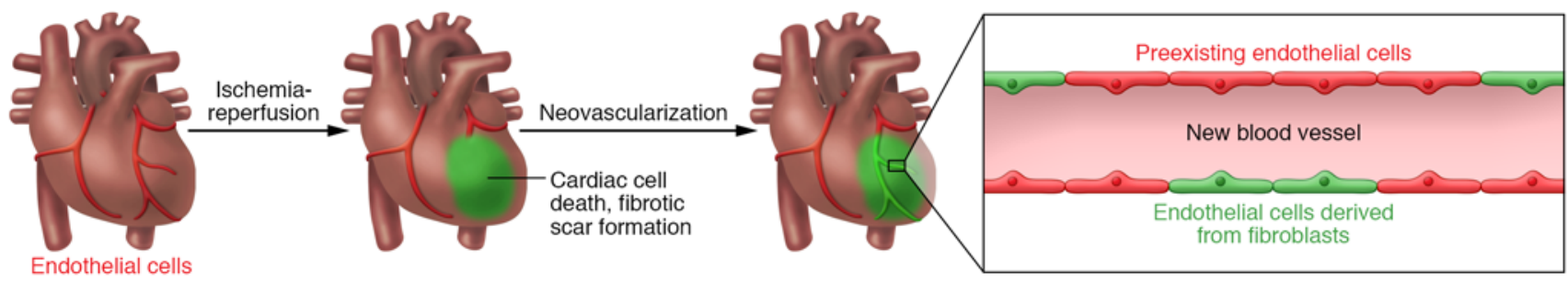

Genetically labeled cardiac fibroblasts

B

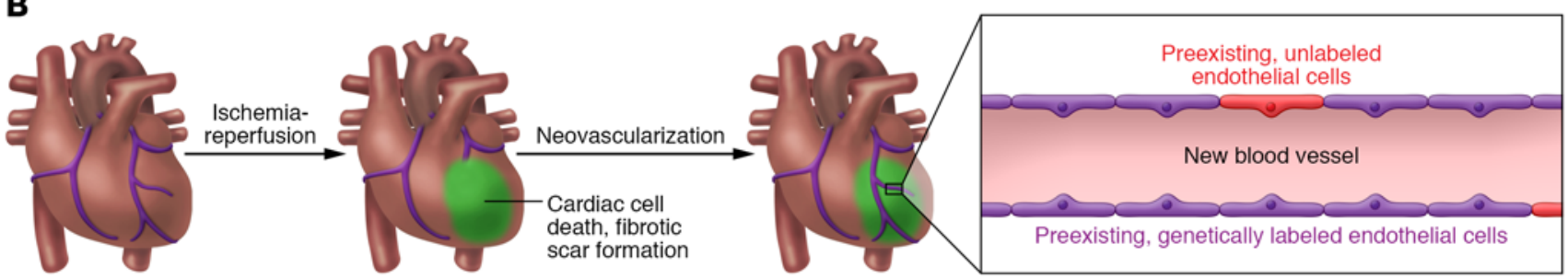

Genetically labeled

endothelial cells

Genetically labeled

cardiac fibroblasts,

mesenchymal cells,

myofibroblasts

Figure 1. Divergent models of the source of new cardiac endothelial cells following injury. (A) Using a transgenic Col1a2-CreER mouse model, Ubil et al. found that preexisting cardiac fibroblasts contribute $30 \%$ to $40 \%$ of new endothelial cells following ischemia-reperfusion injury. (B) In this issue, He et al. performed lineage-tracing and dilution experiments in multiple mouse models to characterize the source of new endothelium following ischemia-reperfusion. Surprisingly, Col1a2-, Pdgfra-, or Tcf21-expressing fibroblasts, Sox9-expressing mesenchymal cells, and Postn-expressing myofibroblasts do not contribute to new coronary endothelial cells following injury. However, preexisting Cdh5-, Apln-, or Fabp4-expressing endothelial cells were a major source of new endothelial cells.

leagues attempted to replicate the experiments of Ubil et al. by treating Colla2-CreER R26R-tdTomato mice with tamoxifen for ten days. Two weeks after the last injection, mice were subjected to ischemia-reperfusion. However, unlike Ubil et al., He et al. did not find any labeled fibroblasts that had converted to endothelial cells (Figure 1B). These discrepant results were striking, leading $\mathrm{He}$ et al. to further investigate. As the Colla2 enhancer/promoter element in the transgenic Col1a2-CreER mouse is inserted into an uncharacterized genomic locus, it is possible that the expression level or efficiency of the Cre transgene has become attenuated over time. To address this possibility, He et al. generated a mouse strain in which $C r e E R$ was inserted directly into the translational stop codon of Colla2 locus. As in the transgenic Col1a2-CreER strain, no endothelial cells could be found among descendants of Col1a2-expressing fibroblasts after injury in the newly derived strain. Future studies by other labs will be needed to independently confirm the efficiency of cardiac fibroblast labeling in the Colla2-CreER knockin mouse line developed by $\mathrm{He}$ et al.
Studies of cardiac fibroblasts have long been complicated by the lack of a single, specific marker for the entire population (7). He et al. addressed this limitation by employing multiple strains of knockin mice to label distinct subpopulations of cardiac fibroblasts. In addition to the Col1a2-CreER knockin mice, $\mathrm{He}$ et al. also generated a mouse strain with an inducible Dre recombinase knocked into the endogenous Pdgfra gene, which is expressed by cardiac fibroblasts. Evaluation of Pdgfra-DreER mice along with strains that allowed inducible labeling of cells expressing Sox9 (mesenchymal cells), transcription factor 21 (Tcf21, fibroblasts), or periostin (Postn, myofibroblasts) revealed that less than $0.05 \%$ of lineagelabeled fibroblasts and myofibroblasts gave rise to PECAM-expressing endothelial cells (Figure 1B). Together, these results suggest that cardiac fibroblasts expressing these markers do not notably contribute to the generation of new endothelium following ischemia-reperfusion injury. Of note, Ubil et al. reported that endothelial cells derived from MEndoT after cardiac injury do not express PECAM, raising the possibility that the two groups could be assaying different sets of cells (9).

$\mathrm{He}$ et al. also performed a lineage dilution experiment to determine whether the proportions of labeled, preexisting cardiac endothelial cells expressing cadherin 5 (Cdh5), apelin (Apln), and fatty acid-binding protein 4 (Fabp4) are reduced in the heart after cardiac injury. A marked dilution of the fraction of labeled endothelial cells would suggest that new endothelial cells are derived from a nonendothelial source; however, most, if not all, cells within the new endothelium at the injury site were descendants of preexisting endothelial cells (Figure 1B). Additionally, $\mathrm{He}$ and colleagues provide evidence that endothelial cell proliferation is a potential mechanism for regenerating damaged endothelium. While lineage dilution experiments may not be sensitive enough to detect rare contributions from alternate cellular sources, these data suggest that fibroblasts are unlikely to contribute to a significant fraction of endothelial cells after cardiac injury. 
Thus, similarly to the mechanism of vasculogenesis during mouse development and in regenerating zebrafish, preexisting endothelial cells contribute to a majority of cells within the new endothelium following cardiac injury in mice (12).

\section{Future perspectives}

The current study by $\mathrm{He}$ et al. establishes a role for preexisting vascular endothelial cells in the formation of new coronary endothelium following ischemia-reperfusion injury. While these findings suggest that novel methods for revascularization should target existing vessels, a number of key issues remain. First, even though $\mathrm{He}$ et al. found new endothelial cell formation following ischemia-reperfusion injury, the speed and degree of neovascularization are generally insufficient to protect or rescue the damaged myocardium following acute myocardial infarction. Thus, identifying factors that modulate the neovascularization process will be needed. Second, while a majority of new endothelial cells appear to be derived from preexisting endothelial cells, it is unclear whether there is a specific subpopulation of endothelial cells that is responsible for the neovascularization process, especially as the markers used by $\mathrm{He}$ et al. are panendothelial. Single cell lineage tracing using markers specific for endothelial cell subtypes (e.g., arterial, venous, endocardial, etc.) or single cell transcriptional profiling of regenerating vasculature may be instructive for further defining the cellular sources of the new endothelium. Finally, although $\mathrm{He}$ et al. provide evidence for preexisting endothelial cell proliferation, it is not clear how proliferation and patterning after injury are regulated. Nonetheless, this added clarification of the cellular origins of coronary neovascularization by $\mathrm{He}$ et al. is an important first step toward further improving revascularization strategies.

\section{Acknowledgments}

$\mathrm{RK}$ is supported by an NIH Mentored Clinical Scientist Award, K08-HL116485, the Walker P. Inman Endowment, and the Edna and Fred L. Mandel, Jr. Foundation. SMW is supported by an NIH Director's Pioneer Award (DP1 LM012179-02), the NHLBI Progenitor Cell Biology Consortium (U01 HL099776), an American Heart Association Established Investigator Award (17EIA33410923), and an Endowed Faculty Scholar Award of the Lucile Packard Foundation for Children and Child Health Research Institute at Stanford.

Address correspondence to: Ravi Karra, Box 3126, Duke University Medical Center, Durham, North Carolina 27710, USA. Phone: 919.684.2449; Email: ravi. karra@duke.edu. Or to: Sean M. Wu, Room G1120A, Lokey Stem Cell Building, 265 Campus Dr., Stanford, California 94305, USA. Phone: 650.724.4498; Email: smwu@stanford.edu.
1. Benjamin EJ, et al. Heart disease and stroke statistics - 2017 update: a report from the American Heart Association. Circulation. 2017;135(10):e146-e603.

2. Red-Horse K, Ueno H, Weissman IL, Krasnow MA. Coronary arteries form by developmental reprogramming of venous cells. Nature. 2010;464(7288):549-553.

3. Wu B, et al. Endocardial cells form the coronary arteries by angiogenesis through myocardial-endocardial VEGF signaling. Cell. 2012;151(5):1083-1096.

4. Katz TC, et al. Distinct compartments of the proepicardial organ give rise to coronary vascular endothelial cells. Dev Cell. 2012;22(3):639-650.

5. Tian X, et al. Vessel formation. De novo formation of a distinct coronary vascular population in neonatal heart. Science. 2014;345(6192):90-94.

6. Combs MD, Yutzey KE. Heart valve development: regulatory networks in development and disease. Circ Res. 2009;105(5):408-421.

7. Moore-Morris T, et al. Resident fibroblast lineages mediate pressure overload-induced cardiac fibrosis. J Clin Invest. 2014;124(7):2921-2934.

8. Zeisberg EM, et al. Endothelial-to-mesenchymal transition contributes to cardiac fibrosis. Nat Med. 2007;13(8):952-961.

9. Ubil E, et al. Mesenchymal-endothelial transition contributes to cardiac neovascularization. Nature. 2014;514(7524):585-590.

10. Zheng B, Zhang Z, Black CM, de Crombrugghe B, Denton CP. Ligand-dependent genetic recombination in fibroblasts: a potentially powerful technique for investigating gene function in fibrosis. Am J Pathol. 2002;160(5):1609-1617.

11. He L, et al. Preexisting endothelial cells mediate cardiac neovascularization after injury. J Clin Invest. 2017;127(8):2968-2981.

12. Zhao L, et al. Notch signaling regulates cardiomyocyte proliferation during zebrafish heart regeneration. Proc Natl Acad Sci US A. 2014;111(4):1403-1408. 